\title{
Research Progress on the Relationship between Vascular Aging and Hypertension
}

\author{
Zeyu Si ${ }^{*}$, Xiaoxuan Fan ${ }^{2}$ \\ ${ }^{1}$ The First Clinical Medical College of Shaanxi University of Traditional Chinese Medicine, Xianyang 712000, Shaanxi \\ Province, China \\ ${ }^{2}$ The Affiliated Hospital of Shaanxi University of Chinese Medicine, Xianyang 712000, Shaanxi Province, China \\ *Corresponding author: Zeyu Si, $3286120051 @ q q . c o m$

\begin{abstract}
Vascular aging refers to the structural and functional changes of the arterial wall with age. Vascular aging plays an important role in elderly diseases, such as hypertension. Therefore, the relationship between vascular aging and hypertension has attracted extensive attention. This article mainly reviews the mechanism of vascular aging and its influence on hypertension, so as to provide new ideas and directions for the research and prevention of hypertension.
\end{abstract}

Keywords: Vascular aging; Hypertension; Mechanism

Publication date: November 2021; Online publication: November 30, 2021

\section{Introduction}

Vascular aging has an important impact on senile diseases, which is mainly reflected in the changes of the structure and function of vascular cell wall, such as vascular wall thickening, vascular calcification, and arterial blood pressure changes ${ }^{[1,2]}$. Hypertension is an epidemic disease, with an increasing incidence in the elderly ${ }^{[3]}$. Therefore, this paper focuses on the relationship between vascular aging and hypertension.

\section{Main physiological changes caused by vascular aging}

\subsection{Vascular calcification}

Vascular calcification refers to ectopic calcium salt deposition at the vascular site. It is usually considered as a phenotype of vascular aging. It is one of the independent risk factors to evaluate the degree of vascular aging and predict the occurrence of cardiovascular events ${ }^{[4]}$. Vascular calcification can be divided into medial calcification and intimal calcification according to the location of calcified plaque formation and development ${ }^{[5]}$.

Medial calcification begins at the inner elastic membrane gap, which then extends to the medial membrane, accompanied by aging and osteogenic differentiation of smooth muscle cells ${ }^{[4]}$. Recent studies have shown that many microRNAs also play a crucial role in the aging and osteogenic transformation process of smooth muscle cells ${ }^{[6]}$. Intimal calcification is a process in which altered vascular cells promote the deposition and development of calcium as well as phosphorus crystals in the lipid necrotic nuclei of atherosclerotic plaques, stimulated by lipid accumulation, oxidative stress, inflammatory necrosis, and other factors along with the progression of atherosclerotic plaques ${ }^{[7]}$.

Experimental studies ${ }^{[8]}$ have shown that the increase of calcium or phosphate levels induce smooth muscle cells to convert to osteogenic phenotype, enabling cells to gain the characteristics of chondrocytes and osteoblasts. Osteoblast smooth muscle cells have shown increased expression of osteogenic markers 
but decreased expression of calcification inhibitor protein. Smooth muscle cells exposed to high calcium levels have shown intracellular calcium overload, which may induce microcalcification and eventually lead to large calcification, resulting in vascular stiffness and high blood pressure.

\subsection{Vascular inflammation}

Angiotensin II related inflammatory signaling cascades are the key mechanisms of aging. These signaling cascades affect the phenotype of vascular cells in autocrine, paracrine, and juxtacrine ways. With aging, the phenotypes of endothelial cells and vascular smooth muscle cells change ${ }^{[9]}$.

Angiotensin II and reactive oxygen species induce telomere shortening or mitochondrial DNA damage of endothelial cells and trigger endothelial cell aging, which is characterized by endothelial cell apoptosis and aging ${ }^{[10]}$. The increased production of advanced glycation end products (AGEs) in arterial extracellular matrix (ECM) can affect the cross-linking between collagens. High levels of AGEs can be observed in aging arterial walls. The increase of AGEs is an important molecular mechanism of age-related

arteriosclerosis and vascular inflammation, which is manifested as arterial glycosylation ${ }^{[11]}$. Amyloid deposition is one of the important characteristics of inflammatory arterial aging. In the amyloid complex, both medin and MFG-E8 bind with elastin and regulate its elasticity. MFG-E8 and medin are potential factors of age-related arterial inflammation and sclerosis, presenting as arterial amyloidosis eventually ${ }^{[12]}$.

\section{Relationship between vascular aging and hypertension}

Hypertension is a complex disease caused by multiple organ dysfunction. Under physiological conditions, vascular endothelial cells activate soluble guanylate cyclase in vascular smooth muscle cells (VSMCs) by continuously releasing nitric oxide (NO), which relaxes the vascular smooth muscle, resulting in vasodilation and the regulation of blood pressure, while the regenerated endothelium partially loses the ability to release NO. Hypertension can cause endothelial injury, reduce the synthesis and release of NO by endothelial cells, as well as further aggravate endothelial injury, forming a vicious circle.

In hypertension, calcium treatment is disturbed. The increased intracellular calcium induces the activation of phospholipase C-coupled receptors, resulting in the production of second messengers and triggering cytokines, ROS, miRNA, as well as cell-derived extracellular vesicles (EV) ${ }^{[13]}$. The elasticity of the vascular system decreases, leading to the increase of blood pressure and the hardening of VSMCs ${ }^{[14]}$. In short, vascular calcification directly or indirectly leads to arteriosclerosis and ultimately hypertension [15].

In addition, AGEs can promote platelet aggregation, reduce vascular elasticity and compliance, as well as accelerate the process of arteriosclerosis by changing the composition of sugar matrix. Hypertension can damage vascular endothelial cells, stimulate smooth muscle to produce a large amount of matrix metalloproteinase (MMP)-9, degrade extracellular matrix, and lead to vascular remodeling ${ }^{[16]}$.

\section{Conclusion}

In conclusion, hypertension can cause vascular damage by affecting cytokines and metabolites, which accelerates vascular aging. This provides a basis for the early intervention of vascular aging. This paper introduces the main mechanism of vascular aging and its correlation with hypertension, so as to provide new ideas and directions for the research and prevention of hypertension.

\section{Disclosure statement}

The authors declare that there is no conflict of interest. 


\section{References}

[1] Tao L, 2011, Study on the Mechanism of TCM Nourishing Qi and Promoting Blood Circulation Delaying Aging and Vascular Aging Induced by Hypertension. China Academy of Chinese Medical Sciences.

[2] Wang Y, 2012, Study on the Correlation between Hypertension and Vascular Aging. Jilin Medical Journal, 33(24): 5185.

[3] Buford TW, 2016, Hypertension and Aging. Ageing Res Rev, 26: 96-111. DOI: 10.1016/j.arr.2016.01.007

[4] Lanzer P, Boehm M, Sorribas V, et al., 2014, Medial Vascular Calcification Revisited: Review and Perspectives. Eur Heart J, 35(23): 1515-1525. DOI: 10.1093/eurheartj/ehul63

[5] Zhang L, Zhang C, 2016, Vascular Calcification and Vascular Aging. Chinese Journal of Geriatrics, 35(10): 1046-1050.

[6] Leopold JA, 2014, MicroRNAs Regulate Vascular Medial Calcification. Cells, 3(4): 963-980. DOI: 10.3390/cells3040963

[7] Otsuka F, Sakakura K, Yahagi K, et al., 2014, Has Our Understanding of Calcification in Human Coronary Atherosclerosis Progressed? Arterioscler Thromb Vasc Biol, 34(4): 724-736. DOI: 10.1161/ATVBAHA.113.302642

[8] Jaminon A, Reesink K, Kroon A, et al., 2019, The Role of Vascular Smooth Muscle Cells in Arterial Remodeling: Focus on Calcification-Related Processes. Int J Mol Sci, 20(22): 5694. DOI: 10.3390/ijms20225694

[9] Zhang L, Wang Y, Liu Y, et al., 2016, Inflammation and Vascular Aging. Chinese Journal of Geriatrics, 35(10): 1038-1041.

[10] Wang M, Monticone RE, Lakatta EG, 2014, Proinflammation of Aging Central Arteries: A Mini Review. Gerontology, 60(6): 519-529. DOI: 10.1159/000362548

[11] Wang M, Jiang L, Monticone RE, et al., 2014, Proinflammation: The Key to Arterial Aging. Trends Endocrinol Metab, 25(2): 72-79. DOI: 10.1016/j.tem.2013.10.002

[12] Wang M, Khazan B, Lakatta EG, 2010, Central Arterial Aging and Angiotensin II Signaling. Curr Hypertens Rev, 6(4): 266-281. DOI: 10.2174/157340210793611668

[13] Touyz RM, Alves-Lopes R, Rios FJ, et al., 2018, Vascular Smooth Muscle Contraction in Hypertension. Cardiovasc Res, 114: 529-539. DOI: 10.1093/cvr/cvy023

[14] Zhu Y, He L, Qu J, et al., 2018, Regulation of Vascular Smooth Muscle Cell Stiffness and Adhesion by $[\mathrm{Ca} 2+] \mathrm{i}$ : An Atomic Force Microscopy-Based Study. Microsc Microanal, 24: 708-712. DOI: $10.1017 / \mathrm{S} 1431927618015519$

[15] Sehgel NL, Zhu Y, Sun Z, et al., 2013, Increased Vascular Smooth Muscle Cell Stiffness: A Novel Mechanism for Aortic Stiffness in Hypertension. Am J Physiol Heart Circ Physiol, 305: H1281-H1287. DOI: 10.1152/ajpheart.00232.2013

[16] Guo H, Song W, Zhang M, et al., 2013, Study on the Correlation between Essential Hypertension and Vascular Aging. Practical Preventive Medicine, 20(03): 370-371. 